\title{
A New Strategy To Stabilize Oxytocin in Aqueous Solutions: II. Suppression of Cysteine-Mediated Intermolecular Reactions by a Combination of Divalent Metal lons and Citrate
}

\author{
Christina Avanti, ${ }^{* \dagger}$ Hjalmar P. Permentier, ${ }^{\ddagger}$ Annie van Dam, ${ }^{\ddagger}$ Robert Poole, ${ }^{\S}$ Wim Jiskoot, ${ }^{\S}$
}

Henderik W. Frijlink, ${ }^{\dagger}$ and Wouter L. J. Hinrichs ${ }^{\dagger}$

${ }^{\dagger}$ Department of Pharmaceutical Technology \& Biopharmacy and ${ }^{\ddagger}$ Mass Spectrometry Core Facility, University of Groningen, Groningen, The Netherlands

${ }^{\S}$ Division of Drug Delivery Technology, Leiden/Amsterdam Center for Drug Research, Leiden University, Leiden, The Netherlands

Supporting Information

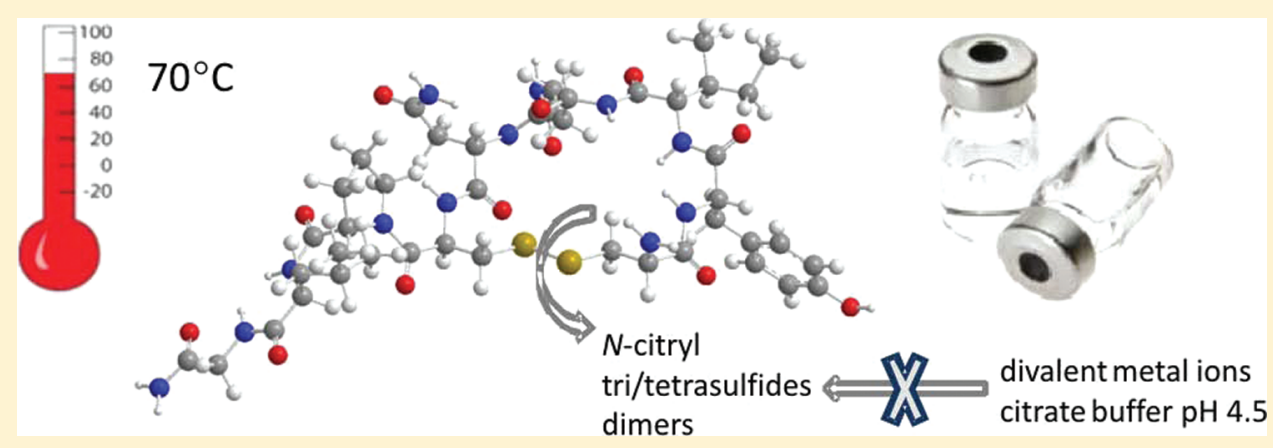

ABSTRACT: A series of studies have been conducted to develop a heat-stable liquid oxytocin formulation. Oxytocin degradation products have been identified including citrate adducts formed in a formulation with citrate buffer. In a more recent study we have found that divalent metal salts in combination with citrate buffer strongly stabilize oxytocin in aqueous solutions (Avanti, C.; et al. AAPS J. 2011, 13, 284-290). The aim of the present investigation was to identify various degradation products of oxytocin in citrate-buffered solution after thermal stress at a temperature of $70{ }^{\circ} \mathrm{C}$ for 5 days and the changes in degradation pattern in the presence of divalent metal ions. Degradation products of oxytocin in the citrate buffer formulation with and without divalent metal ions were analyzed using liquid chromatography-mass spectrometry/mass spectrometry (LC-MS/MS). In the presence of divalent metal ions, almost all degradation products, in particular citrate adduct, tri- and tetrasulfides, and dimers, were greatly reduced in intensity. No significant difference in the stabilizing effect was found among the divalent metal ions $\mathrm{Ca}^{2+}, \mathrm{Mg}^{2+}$, and $\mathrm{Zn}^{2+}$. The suppressed degradation products all involve the cysteine residues. We therefore postulate that cysteine-mediated intermolecular reactions are suppressed by complex formation of the divalent metal ion and citrate with oxytocin, thereby inhibiting the formation of citrate adducts and reactions of the cysteine thiol group in oxytocin.

KEYWORDS: oxytocin, aqueous solution, degradation, citrate buffer, divalent metal ions

\section{INTRODUCTION}

Oxytocin is a neurohypophyseal hormone, which was first discovered by H. H. Dale in 1909. ${ }^{1,2}$ Oxytocin is produced by neurons of the posterior lobe of the hypophysis and pulsatively released into the periphery. In clinical practice, oxytocin has been prescribed primarily for labor induction and augmentation, control of postpartum hemorrhage and uterine hypotonicity in the third stage of labor. ${ }^{3,4}$ Oxytocin is commonly administered by intravenous infusion. ${ }^{5}$

The oxytocin structure was elucidated in $1951,{ }^{6-8}$ and the characterization and biosynthesis of oxytocin were reported in 1953 by du Vigneaud. ${ }^{9}$ Oxytocin consists of nine amino acids: cyclo- $\left(\mathrm{Cys}^{1}-\mathrm{Tyr}^{2}-\mathrm{Ile}^{3}-\mathrm{Gln}^{4}-\mathrm{Asn}^{5}-\mathrm{Cys}^{6}\right)-\mathrm{Pro}^{7}-\mathrm{Leu}^{8}-\mathrm{Gly}^{9}-\mathrm{NH}_{2}$ with a disulfide bridge between Cys residues 1 and $6 .^{10,11}$ The primary structure of oxytocin is shown in Figure 1. A major problem of the compound is its intrinsic instability in aqueous formulations. ${ }^{12}$ Recently significant attention was focused on efforts to overcome the instability of oxytocin. ${ }^{13,14} \mathrm{We}$ have conducted several studies with the aim to develop a heat-stable oxytocin formulation.

We identified the main degradation products of oxytocin stressed at a temperature of $70{ }^{\circ} \mathrm{C}$ in various buffers, $\mathrm{pH}$ values and storage time, ${ }^{13}$ as well as citryl oxytocin in citrate-buffered formulations. ${ }^{14}$ The degradation reactions and target residues of oxytocin are indicated in Figure 1.

Received: August 4, 2011

Revised: January 9, 2012

Accepted: January 18, 2012

Published: January 18, 2012 


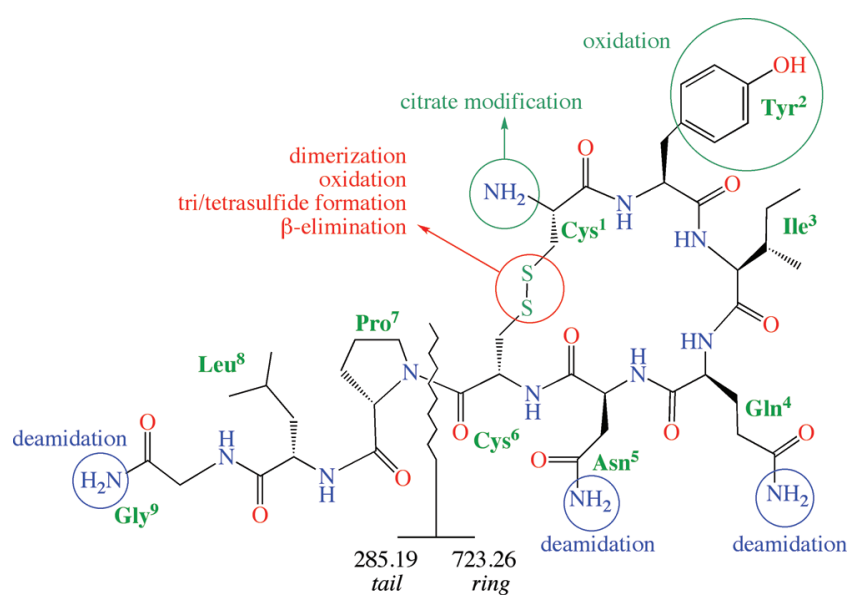

Figure 1. Molecular structure of oxytocin with its ring and tail fragment ions formed upon MS/MS fragmentation.

In a recent publication we described that formulations containing divalent metal salts in combination with citrate buffer strongly stabilize oxytocin in aqueous solutions. ${ }^{15}$ However, the role by which divalent metal ions stabilize oxytocin in citrate buffer and their influence on (inhibition of) formation of degradation products were not elucidated. Therefore, the aim of the present study was to identify by LC-MS/MS the degradation products of oxytocin solutions after thermal stress and to investigate which degradation pathways are suppressed by the formulations containing combinations of divalent metal ions and citrate buffer.

\section{MATERIALS AND METHODS}

Materials. The following materials were used in this study: oxytocin monoacetate powder (Diosynth, Oss, The Netherlands), citric acid, calcium chloride (Riedel-de Haen, Seelze, Germany), magnesium chloride, zinc chloride (Fluka, Steinheim, Germany), sodium hydroxide, acetonitrile, ammonium acetate (Merck, Darmstadt, Germany), DL-dithiothreitol and ammonium bicarbonate (Sigma-Aldrich Chemie Gmbh, Steinheim, Germany).

Formulation and Stability Study. Three independent batches of each of the four formulations given in Table 1 were

\section{Table 1. The Composition of Oxytocin Liquid Formulation}

$\begin{array}{cccc}\text { formulation }^{a} & \text { oxytocin } & \text { metal ion } & \text { buffer } \\ \text { OCB } & 0.1 \mathrm{mM} & & \text { citrate } 10 \mathrm{mM} \\ \text { OCBCa } & 0.1 \mathrm{mM} & \mathrm{Ca}^{2+} 10 \mathrm{mM} & \text { citrate } 10 \mathrm{mM} \\ \text { OCBMg } & 0.1 \mathrm{mM} & \mathrm{Mg}^{2+} 10 \mathrm{mM} & \text { citrate } 10 \mathrm{mM} \\ \text { OCBZn } & 0.1 \mathrm{mM} & \mathrm{Zn}^{2+} 10 \mathrm{mM} & \text { citrate } 10 \mathrm{mM}\end{array}$

${ }^{a} \mathrm{OCB}=$ oxytocin in the absence of divalent metal ions in $10 \mathrm{mM}$ citrate-buffered solution at $\mathrm{pH} 4.5$. OCBCa $=$ oxytocin in the presence of $\mathrm{Ca}^{2+}$ in $10 \mathrm{mM}$ citrate-buffered solution at $\mathrm{pH} 4.5$. OCBMg = oxytocin in the presence of $\mathrm{Mg}^{2+}$ in $10 \mathrm{mM}$ citrate-buffered solution at $\mathrm{pH}$ 4.5. OCBZn = oxytocin in the presence of $\mathrm{Zn}^{2+}$ in $10 \mathrm{mM}$ citratebuffered solution at $\mathrm{pH} 4.5$.

prepared. The concentration of the citrate buffer was $10 \mathrm{mM}$, and the $\mathrm{pH}$ was adjusted to 4.5. All metal ion $\left(\mathrm{M}^{2+}\right)$ solutions were prepared using their respective chloride salts at concentrations of $10 \mathrm{mM}\left(\mathrm{MCl}_{2}\right)$. The solutions were stored for 5 days protected from light at either 4 or $70{ }^{\circ} \mathrm{C}$. Prior to analysis, the samples were diluted 10 -fold with water.
Reversed-Phase High Performance Liquid Chromatography. HPLC was performed using a Shimadzu LC system, consisting of LC-20AD gradient pumps and a SIL-20AC autosampler. Chromatographic separation was achieved on an Alltima C18 column $(2.1 \times 150 \mathrm{~mm} 5 \mu \mathrm{m}$, Grace Davison Discovery Sciences). The injection volume was $50 \mu \mathrm{L}$. Elution was performed by a linear gradient from $5 \%$ to $60 \%$ B in $30 \mathrm{~min}$, followed by an increase to $90 \% \mathrm{~B}$ in $1 \mathrm{~min}$, where it was kept $4 \mathrm{~min}$, after which it returned to the starting conditions. Eluent A was $95 \%$ water $/ 5 \%$ acetonitrile $(\mathrm{AcN})$, and eluent B was $5 \%$ water $/ 95 \% \mathrm{AcN}$, both containing $0.05 \% \mathrm{v} / \mathrm{v}$ acetic acid and $10 \mathrm{mM}$ ammonium acetate. The flow rate was $0.2 \mathrm{~mL} / \mathrm{min}$. The UV signal was recorded at $220 \mathrm{~nm}$.

Liquid Chromatography-Mass Spectrometry. The HPLC system was coupled to an API 3000 triple-quadrupole mass spectrometer (Applied Biosystems/MDS Sciex) via a turbo ion spray source. The ionization was performed by electrospray in the positive mode. Full scan spectra were recorded at a scan rate of $2 \mathrm{~s}$ from $\mathrm{m} / z 500$ to 1300 and a step size of $0.2 \mathrm{amu}$ with a declustering potential (DP) of $40 \mathrm{~V}$ and a focusing potential (FP) of $250 \mathrm{~V}$. Product ion scans were acquired in specified time windows with a DP of $60 \mathrm{~V}$, a FP of $300 \mathrm{~V}$, a collision energy of $40 \mathrm{~V}$ and a collision cell exit potential of $20 \mathrm{~V}$. Data acquisition and processing was performed using Analyst version 1.4.2 and 1.5 software (Applied Biosystems/ MDS Sciex).

LC-MS/MS were used to identify the oxytocin degradation products observed by RP-HPLC. The analyses were carried out on purified fractions corresponding to each of the major degradation peaks for oxytocin in citrate buffer formulation without divalent metal salts as well as by LC-MS for the formulation with and without divalent metal salts.

Reduction of disulfide bonds was performed by adding $10 \mathrm{mM}$ DL-dithiothreitol (DTT) in $200 \mathrm{mM}$ ammonium carbonate. The samples were analyzed after an incubation time of at least $10 \mathrm{~min}$ at room temperature.

\section{RESULTS}

Degradation Products of Oxytocin in Citrate Buffer with and without Divalent Metal lons. Assignment of degradation products was done based on the $\mathrm{m} / z$ value of each compound from the LC-MS data, using known assignments and retention time order from previous studies. ${ }^{13,14} \mathrm{MS} / \mathrm{MS}$ data were used for the more intense peaks to confirm the identification and to determine in which part of the molecule the modification had occurred.

The highest degradation product intensities were found in the formulation without divalent metal salts stressed at $70{ }^{\circ} \mathrm{C}$ for 5 days. The HPLC profiles based on ultraviolet (UV) absorbance at $220 \mathrm{~nm}$ and the total ion current (TIC) of mass spectra with $m / z$ between 900 and 1200 are shown in Figure 2a and Figure $2 b$, respectively. The UV profile shows 11 main peaks and the TIC 12 main peaks (see labels in Figure $2 \mathrm{~b}$ ). The LC-MS contour plot (Figure 2c) reveals that some peaks contain several molecular species, which cannot be distinguished in the TIC, notably peaks 2 and 3 reveal two compounds at 12.0 and $12.1 \mathrm{~min}$, peaks 10 and 11 reveal two compounds at 16.7 and $16.8 \mathrm{~min}$, and peaks 14 and 15 reveal two compounds at 18.7 and 18.9 min. All 15 assigned molecular species are presented in Table 2, and MS/MS product ion spectra are shown in Figures $\mathrm{S} 1-\mathrm{S} 10$ in the Supporting Information.

The protonated molecular ion of unmodified oxytocin was found at a retention time of $12.6 \mathrm{~min}$. Figure $2 \mathrm{~d}$ shows its 
a

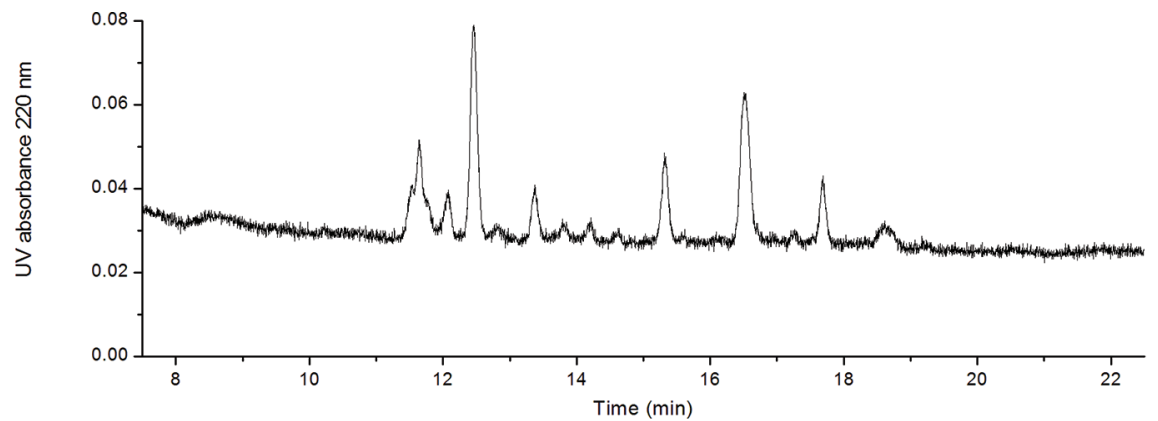

b

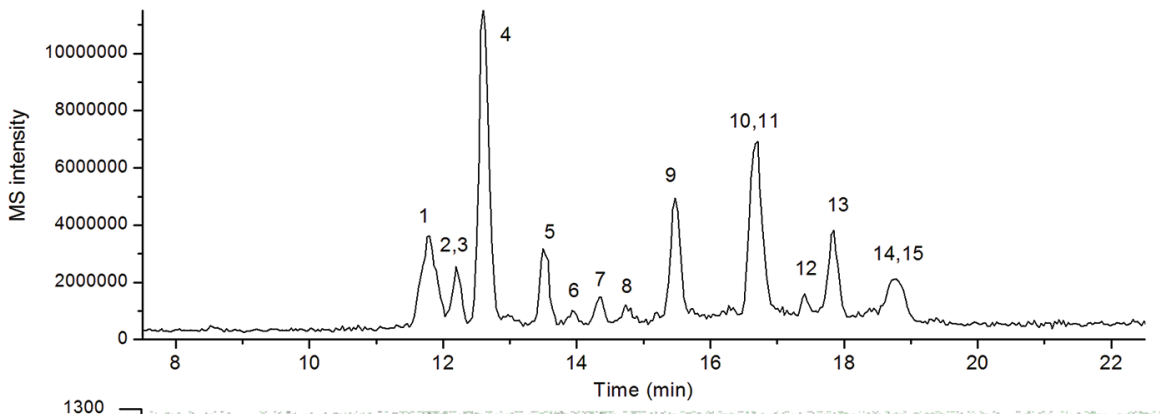

c

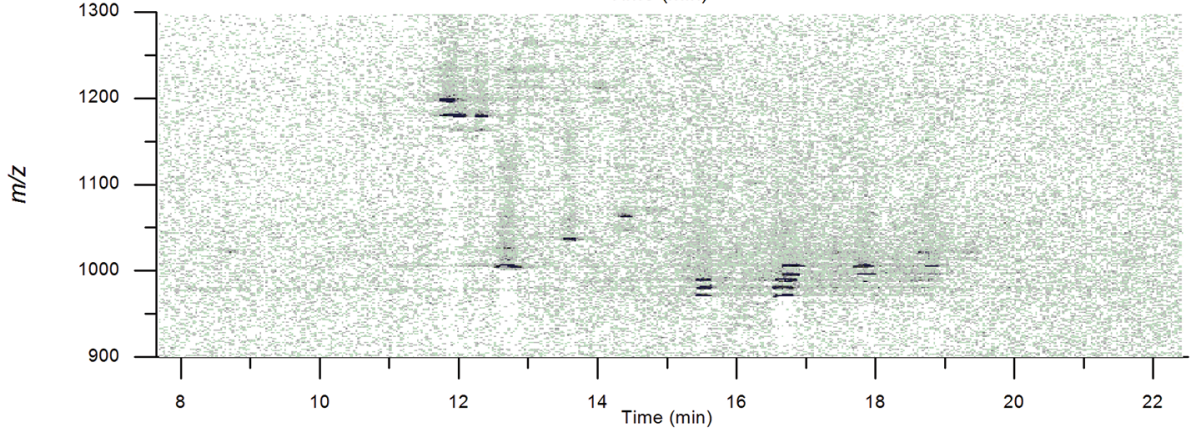

d
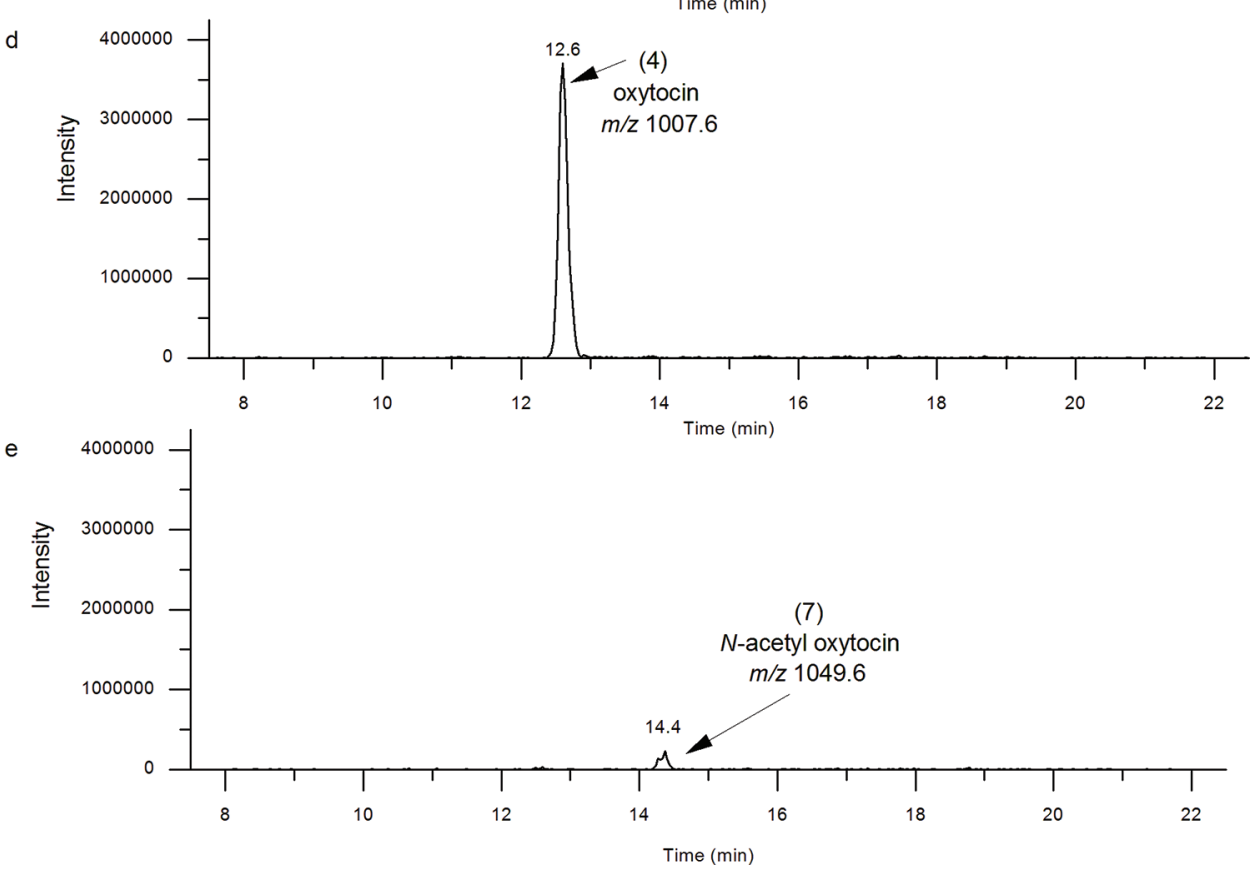

Figure 2. LC-UV trace (a), total ion chromatogram $(\mathrm{m} / \mathrm{z}$ 900-1200) (b), and LC-MS contour plot (c) of stressed oxytocin in citrate buffered solution with the extracted ion current of oxytocin (d) and acetylated oxytocin (e).

extracted ion chromatogram (XIC) at $\mathrm{m} / z$ 1007.6. MS/MS fragmentation of unmodified oxytocin results in fragments of $m / z 723.4$ and 285.2, from the disulfide-linked ring of residues 1-6 and the C-terminal residues 7-9, respectively (Figure 1 and Figure S4 in the Supporting Information).
The degradation peaks eluting before the oxytocin peak (labeled 1 and 2 in Figure $2 b$ ) were identified as amide- and imide-linked $N$-citryl oxytocin with $m / z$ of 1181.8 and 1163.8 , respectively. ${ }^{14}$ The MS/MS spectrum of $m / z 1181.6$ showed that the ring fragment was shifted from $m / z 723.4$ to 897.4 , but 
Table 2. Summary of the Observed Degradation Products of Oxytocin after Stressing at $70{ }^{\circ} \mathrm{C}$ for 5 Days in $10 \mathrm{mM}$ Citrate Buffer

\begin{tabular}{|c|c|c|c|c|}
\hline no. & $t_{\mathrm{R}}(\min )$ & $m / z$ & charge & assignment \\
\hline 1 & 11.8 & 1181.6 & $1+$ & oxytocin $N$-citryl amide \\
\hline 2 & 12.0 & 1008.6 & $1+$ & monodeamidation at $\mathrm{Asn}^{5}$ or $\mathrm{Gln}^{4}$ \\
\hline 3 & 12.1 & 1163.6 & $1+$ & oxytocin $N$-citryl imide \\
\hline 4 & 12.6 & 1007.6 & $1+$ & oxytocin $^{a}$ \\
\hline 5 & 13.5 & 1039.6 & $1+$ & oxytocin trisulfide \\
\hline 6 & 13.9 & 1195.6 & $1+$ & oxytocin trisulfide $N$-citryl imide \\
\hline 7 & 14.4 & 1049.6 & $1+$ & $N$-acetyl oxytocin ${ }^{a}$ \\
\hline 8 & 14.8 & 1071.6 & $1+$ & oxytocin tetrasulfide \\
\hline 9 & 15.5 & 975.6 & $2+$ & dimer 1 \\
\hline 10 & 16.7 & 975.6 & $2+$ & dimer 2 \\
\hline 11 & 16.8 & 1008.6 & $2+$ & dimer 3 \\
\hline 12 & 17.4 & $1024.6^{b}$ & $2+$ & dimer 4 \\
\hline 13 & 17.8 & 1008.6 & $2+$ & dimer 5 \\
\hline 14 & 18.7 & $1024.6^{b}$ & $2+$ & dimer 6 \\
\hline 15 & 18.9 & 1008.6 & $2+$ & dimer 7 \\
\hline
\end{tabular}

${ }^{a}$ No degradation products; compounds were also found in the original oxytocin preparation. ${ }^{b}$ Ammoniated.

the tail fragment was still observed at $m / z 285$.2. It shows that the citrate modification is in the ring fragment and the most likely location is on the N-terminal amine (see Figure 1 and Figure S2 in the Supporting Information). ${ }^{14}$

At a retention time of 12.0 and $12.1 \mathrm{~min}$ (Figure $3 \mathrm{~b}$ and $3 \mathrm{c}$ ), there is overlap of two different compounds (peaks 2 and 3), namely a monodeamidated species of oxytocin with a protonated molecular ion at $m / z 1008.6$ in a very low intensity and dehydrated (imide-linked) $N$-citryl oxytocin with the protonated molecular ion at $m / z$ 1163.6. ${ }^{14}$ The MS/MS spectrum of $m / z$ 1008.6 showed a shift of the ring fragment from $m / z 723.4$ to 724.4, but no shift of the tail fragment mass (Figure S3 in the Supporting Information), which means that the deamidation occurred at $\mathrm{Asn}^{5}$ or $\mathrm{Gln}^{4}{ }^{43}$ The ${ }^{13} \mathrm{C}$ denoted as peak in Figure 3c is the second isotope peak of oxytocin $(\mathrm{m} / z$ 1007.6), which is also found at $\mathrm{m} / z$ 1008.6. The three other peaks in Figure $3 \mathrm{~b}$, at retention times of $16.8,17.8$, and $18.9 \mathrm{~min}$, are the dimer degradation products described in Figure 4c.

A compound at $m / z 1049.6$ was found at a retention time of $14.4 \mathrm{~min}$ (Figure 2e) in every sample including the original oxytocin preparation. The difference of $+42 \mathrm{Da}$ with respect to oxytocin suggests acetylation, presumably at the $\mathrm{N}$-terminal amine (see Figure S6 in the Supporting Information). This acetylation reaction may have occurred during synthesis since oxytocin is supplied as the monoacetate salt.

At a retention time of $13.5 \mathrm{~min}$ a trisulfide degradation product was identified from the $m / z 1039.6$ peak. Upon MS/ MS fragmentation, the ring fragment shifted to $m / z$ 755.4, while the tail fragment mass did not change (see Figure S5 in the Supporting Information). The mass difference of $+32 \mathrm{Da}$ can be assigned to a trisulfide modification in the oxytocin ring. ${ }^{13}$ At a retention time of $13.9 \mathrm{~min}$, a product of $\mathrm{m} / z 1195.6$ was found, which is tentatively identified as the $N$-citryl oxytocin with the trisulfide modification. The formation of a tetrasulfide product $(\mathrm{m} / z$ 1071.6) was found at the retention time of $14.8 \mathrm{~min}$. Molecular structure $\mathrm{N}$-citryl oxytocin, trisulfide and tetrasulfide modification produced from the degradation of stressed oxytocin in citrate buffered solution are shown in Figure 5.
Seven peaks of dimers were evident as doubly charged ions in MS eluting between 15.5 and $18.9 \mathrm{~min}$ (Table 2 and Figure 4). Dimer 1 and 2 had identical masses $(\mathrm{m} / z$ 975.6) and were also observed in a previous study of stressed oxytocin solutions ${ }^{13}$ where they were assigned to the doubly protonated form of a sulfur-linked dimeric oxytocin species that has been doubly deamidated and which has lost two sulfur atoms through $\beta$-elimination. ${ }^{13}$

Identification of dimers is difficult due to the overlapping LC peaks and multiple ion forms (protonated and ammoniated, and doubly charged, Figure 2c). In addition, their MS/MS fragmentation spectra only show the tail fragment of $\mathrm{m} / z 285.2$ (Figures $57-10$ in the Supporting Information) and consequently they are not informative.

In order to distinguish disulfide linked dimers from other types of dimers, we have done the LC-MS analysis on the most degraded solution after disulfide bond reduction with DTT. All monomeric products observed were reduced, as summarized in Table 3, producing oxytocin, oxytocin acetate, and $\mathrm{N}$-citryl amide/imide in the reduced thiol form (Figures S11-S17 in the Supporting Information). Tri- and tetrasulfide forms were also absent after reduction and are expected to have been converted to reduced oxytocin. The product ion spectrum after DTT reduction of $\mathrm{m} / z 993.6$ at a retention time of $16.6 \mathrm{~min}$ (Figure S18 in the Supporting Information) showed that it is a monomer which has undergone $\beta$-elimination at Cys $^{1}$ and deamidation at $\mathrm{Asn}^{5}$ or Gln ${ }^{4}$. This is as evidenced by the presence of an unmodified $y_{4}$ ion and $a b_{6}$ ion (Figure $S 1$ in the Supporting Information) mass of $-33 \mathrm{Da}$ with respect to oxytocin. $\beta$-Elimination leads to a loss of $34 \mathrm{Da}\left(-\mathrm{H}_{2} \mathrm{~S}\right)$, and deamidation increases the mass by $1 \mathrm{Da}$. The presence of this reduced monomer at high intensity confirms the assignment of dimers 1 and 2, which proves that these dimers are disulfidelinked at the unmodified $\mathrm{Cys}^{6}$ residues.

In the LC-MS profile (Figure 6) it was also found that the LC-MS peaks of all dimers had disappeared after reduction. However there were 2 new peaks present at retention times of 17.9 and $18.1 \mathrm{~min}$ with $\mathrm{m} / z 994.0$ and 993.6 respectively, which are doubly charged ion (Figures S19-S20 in the Supporting Information). The presence of dimers after DTT reduction, combined with the observation that all original 7 dimer peaks were absent after reduction, shows that all dimers had at least one disulfide bond and some had an additional thio-ether bond (which may form after $\beta$-elimination and reaction with another Cys residue). Tyrosine-linked dimers were unlikely to be present, since no monomeric tyrosine oxidation products were observed.

Effects of Divalent Metal Ions on Oxytocin's Degradation Profile. Addition of divalent metal salts in the formulation did not result in any additional degradation products compared to samples without metal salts. As expected from our previous study, ${ }^{14}$ it resulted in a significant reduction of most degradation peaks. Figure 7 shows that, of all formulations tested, oxytocin formulated in $10 \mathrm{mM}$ citrate buffer $(\mathrm{pH} \mathrm{4.5)}$ without divalent metal ions (OCB) was most degraded with only approximately $35 \%$ oxytocin recovered after incubation at $70{ }^{\circ} \mathrm{C}$ for 5 days. However, when divalent metal ions were added, the degradation was decelerated and a recovery of about $70 \%$ oxytocin was found, irrespective of the nature of the divalent metal ion, which is in line with our previous study. ${ }^{15}$

From each stressed formulation of oxytocin in citrate buffer in the presence of calcium, magnesium, or zinc ions, we recorded the intensity of each degradation product from their 
a

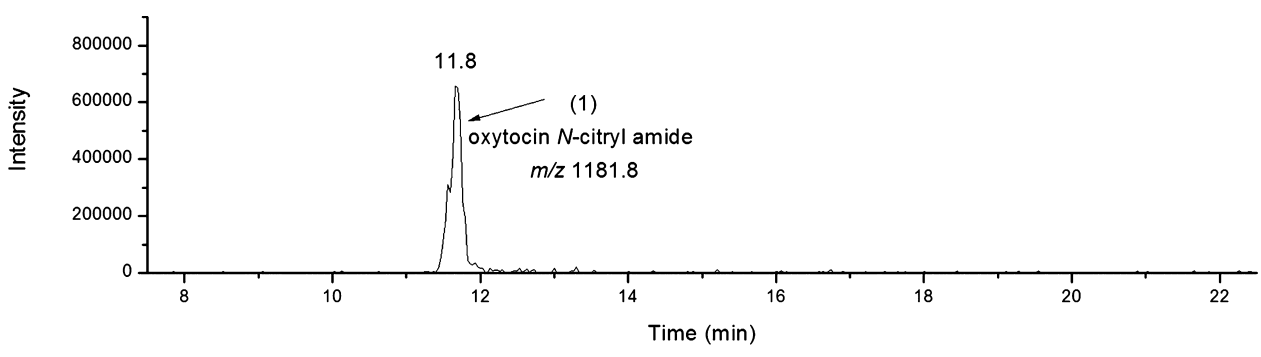

b

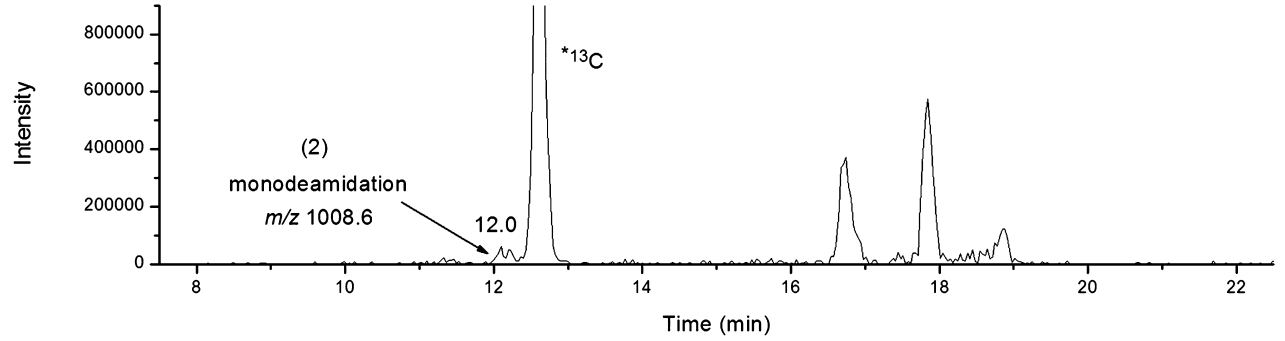

c

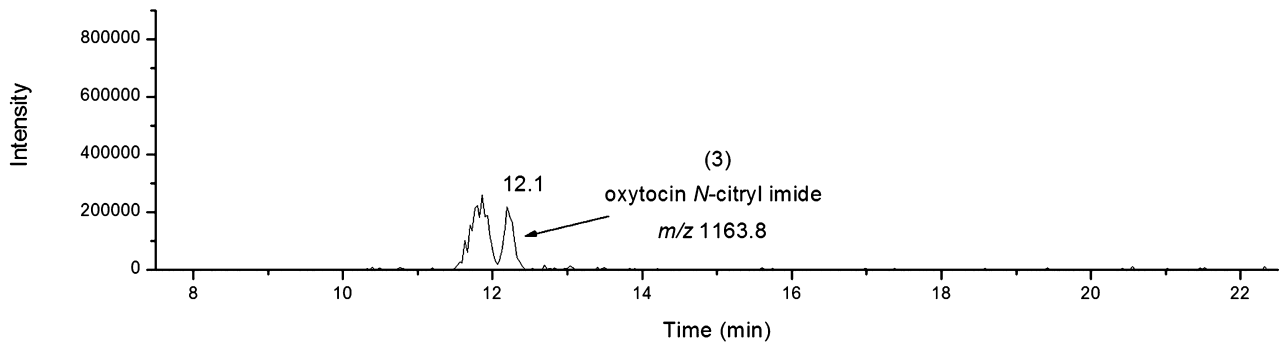

d
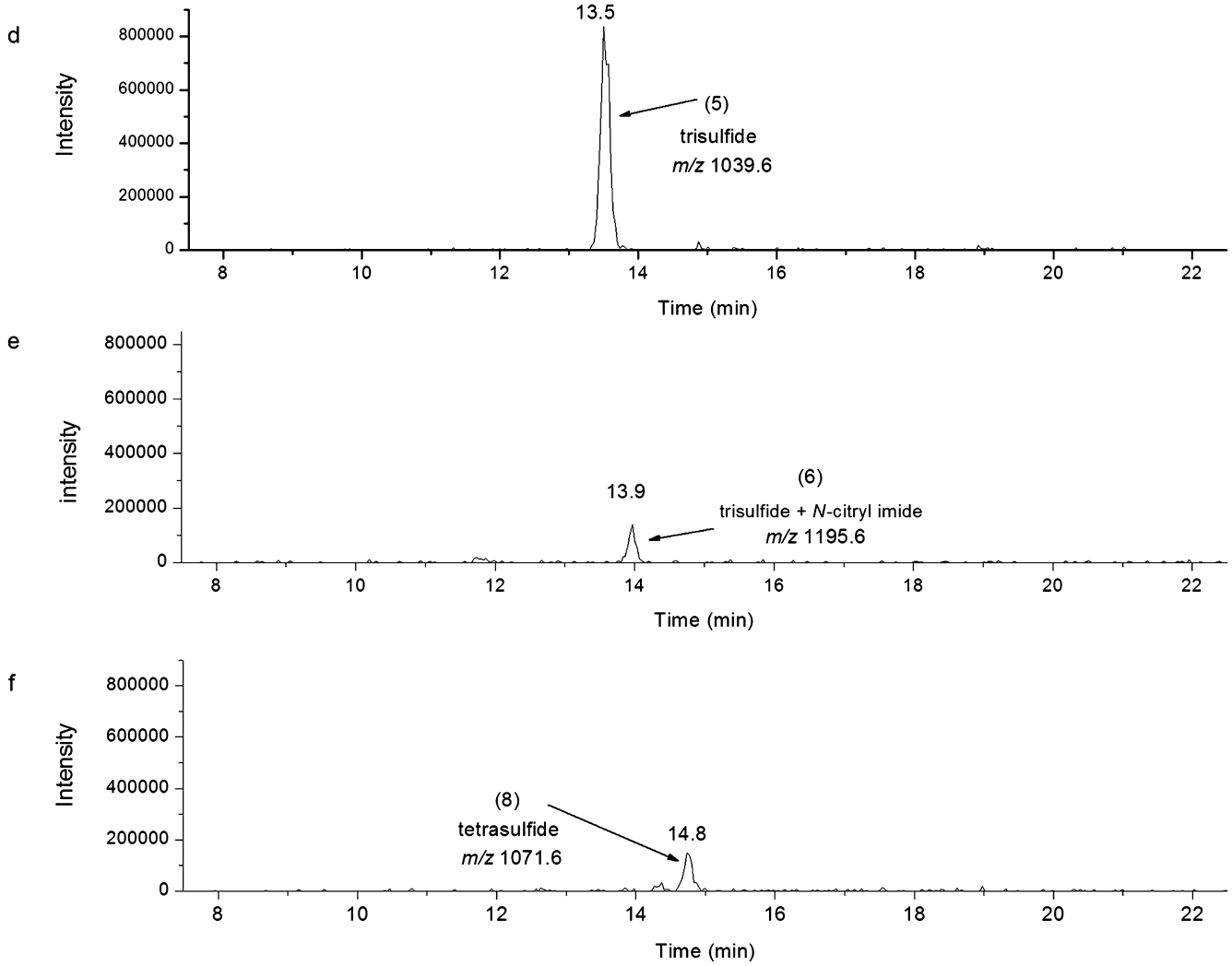

Figure 3. LC-MS extracted ion currents for oxytocin $N$-citryl amide (a) and $N$-citryl imide (b), monodeamidation (c), trisulfide (d), trisulfide $\mathrm{N}$-citryl imide (e), and tetrasulfide products from the degradation of stressed oxytocin in citrate buffered solution.

respective extracted MS ion currents. The relative intensities in percentage of the peak area of each degradation product in the presence of divalent metal ions with respect to that of the same product in the absence of divalent metal ions are listed in Table 4.
Addition of divalent metal ions reduced the peak intensity of almost all degradation products. The formation of the $\mathrm{N}$-citryl oxytocin and the tri- and tetrasulfide was reduced by $20-70 \%$. The formation of the dehydrated $\mathrm{N}$-citryl oxytocin and dimers 
16.7
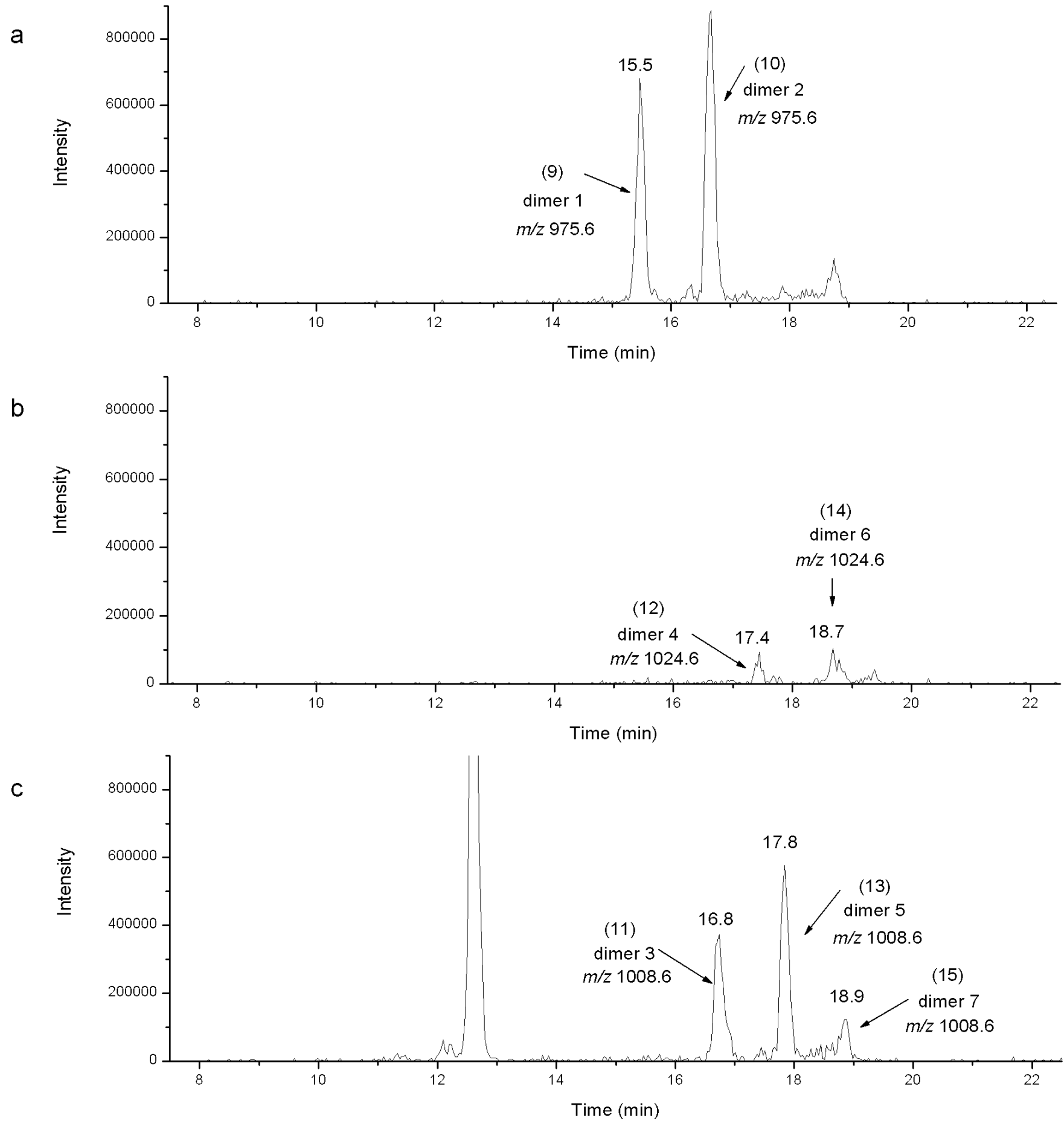

Figure 4. LC-MS extracted ion currents for dimer degradation products.

$1,2,4,5$ was even more strongly reduced, by $90 \%$. No clear reduction of the intensity of the deamidated species $(\mathrm{m} / \mathrm{z}$ 1008.6, retention time $12.1 \mathrm{~min})$ and dimer $3(\mathrm{~m} / z$ 1024.8, retention time $17.4 \mathrm{~min}$ ) was observed, possibly because of their low signal intensity which made peak integration difficult (Figures $3 b$ and $4 b$ ).

The acetylated oxytocin species (peak number 7) appeared to be very stable: no significant difference in its relative intensity was observed either in the presence or in the absence of divalent metal ions. In addition, there is no significant difference of the intensity of this product in unstressed or stressed condition. We conclude that this molecule is not a degradation product formed under stress conditions but, as mentioned above, an impurity of oxytocin formed during synthesis.

\section{DISCUSSION}

Several biological and physicochemical methods have been described to monitor the stability of oxytocin. HPLC with $\mathrm{UV} /$ vis detection is the most frequently used physicochemical method to monitor oxytocin stability. Especially when combined with mass spectrometry (MS) detection, most degradation products can be identified and quantified. ${ }^{16,17}$ Hyphenation of 
(a)

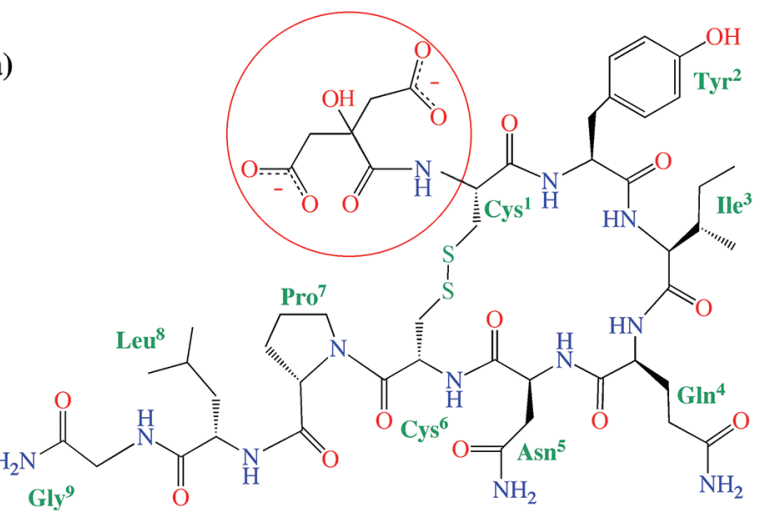

(c)

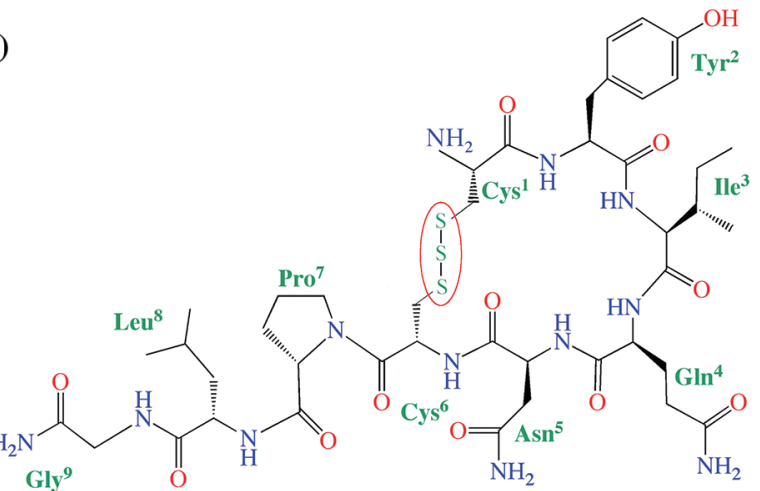

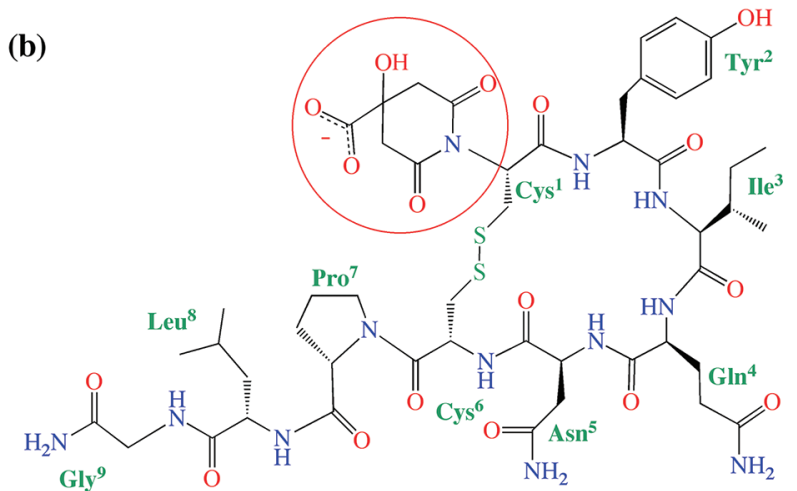

(d)

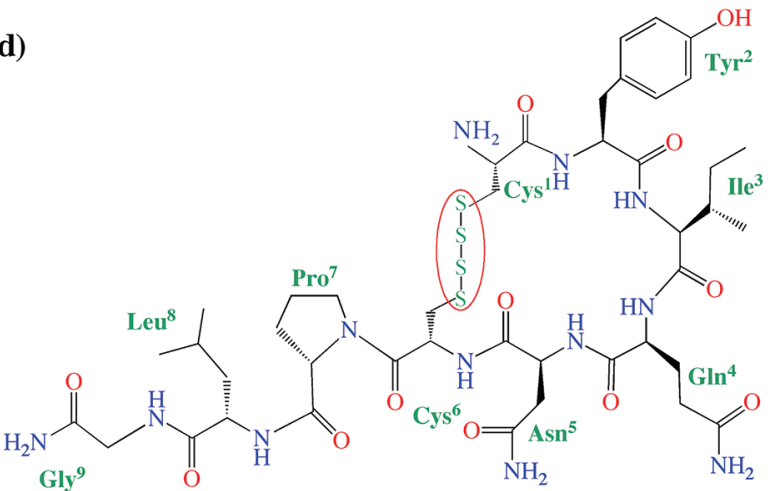

Figure 5. Molecular structures of oxytocin $N$-citryl amide (a), $N$-citryl imide (b), trisulfide (c) and tetrasulfide (d) produced from the degradation of stressed oxytocin in citrate buffered solution.

Table 3. Summary of the Observed Degradation Products of Oxytocin after Stressing at $70{ }^{\circ} \mathrm{C}$ for 5 Days in $10 \mathrm{mM}$ Citrate Buffer Followed by Reduction by DTT $^{a}$

\begin{tabular}{|c|c|c|c|c|}
\hline no. & $\begin{array}{c}t_{\mathrm{R}} \\
(\mathrm{min})\end{array}$ & $m / z$ & charge & assignment \\
\hline a & 12.8 & 1007.4 & $1+$ & oxytocin (disulfide) \\
\hline$b$ & 13.1 & 1200.6 & $1+$ & oxytocin $N$-citryl amide $b$ \\
\hline c & 13.5 & 1009.6 & $1+$ & oxytocin \\
\hline $\mathrm{d}$ & 14.6 & 1182.6 & $1+$ & oxytocin $N$-citryl imide ${ }^{b}$ \\
\hline e & 15.5 & 1068.8 & $1+$ & $N$-acetyl oxytocin ${ }^{b}$ \\
\hline $\mathrm{f}$ & 16.6 & 993.6 & $1+$ & $\begin{array}{l}\beta \text {-elimination at } \mathrm{Cys}^{1}, \text { monodeamidation at } \\
\mathrm{Asn}^{5} \text { or } \mathrm{Gln}{ }^{4 b}\end{array}$ \\
\hline $\mathrm{g}$ & 17.9 & 994.0 & $2+$ & dimer \\
\hline $\mathrm{h}$ & 18.1 & 993.6 & $2+$ & dimer \\
\hline
\end{tabular}

the LC unit to MS via an electrospray ionization (ESI) interface allows sensitive and selective confirmation of degradation products by extracting corresponding ion chromatograms from the recorded total ion current (TIC) ${ }^{14}$ and by using MS/MS for the identification of degradation products. Furthermore, previous studies have demonstrated that LC-MS can be used to monitor the stability of oxytocin in pharmaceutical dosage forms. ${ }^{13,18}$

The degradation of oxytocin in various formulations was analyzed after 5 days of incubation at $70{ }^{\circ} \mathrm{C}$. A temperature of $70{ }^{\circ} \mathrm{C}$ was chosen to ensure the formation of qualitatively similar degradation products as found by Poole et al., who incubated oxytocin in citrate buffered solutions at $70{ }^{\circ} \mathrm{C}$ for 2 days. ${ }^{14}$ The aim of the present study was to investigate the effect of stabilizing divalent metal ions on the degradation profile. In order to observe sufficient amounts for characterization of all

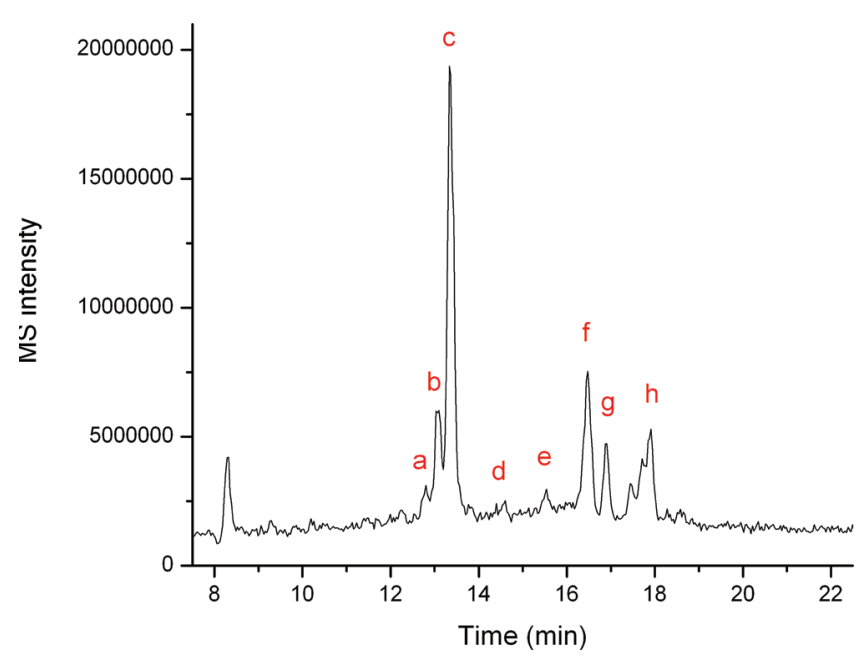

Figure 6. Total ion chromatogram $(m / z$ 900-1200) of stressed oxytocin in citrate buffered solution followed by reduction using DTT.

degradation products in the stabilized formulations, it was necessary to prolong the thermal stress from 2 to 5 days.

The strongly decreased intensity of the dimeric products suggests that formulation with divalent metal ions protects against thiol exchange, hence avoiding dimerization. At low $\mathrm{pH}$, dimerization occurs via thiol exchange in the disulfide bridge, which progresses via a sulfonium cation, which is formed following protonation of the disulfide bridge. ${ }^{19,20}$ This could also explain the ability of these formulations to inhibit the formation of tri/tetrasulfide oxytocin species. 


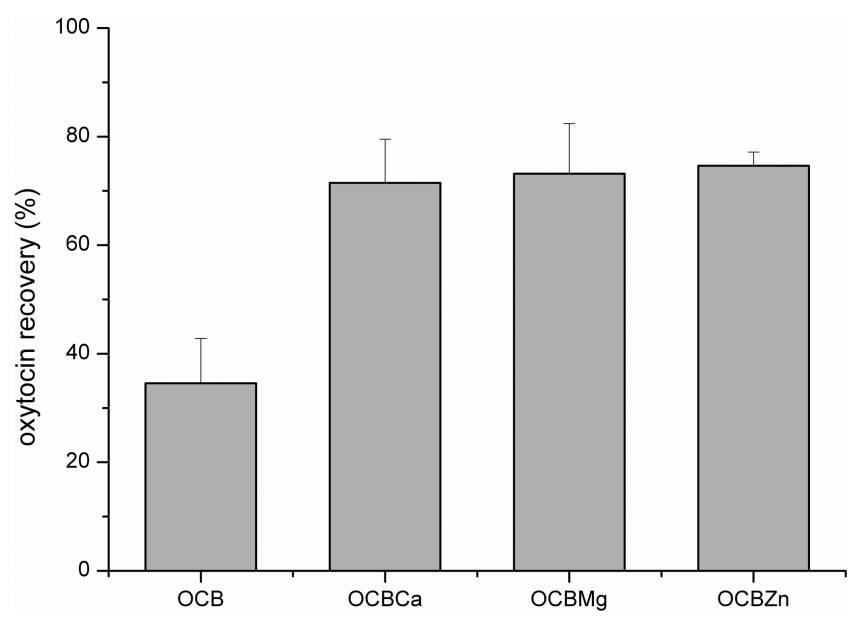

Figure 7. Recovery of oxytocin in the absence (OCB) and presence of $10 \mathrm{mM} \mathrm{Ca}{ }^{2+}$ (OCBCa), $\mathrm{Mg}^{2+}$ (OCBMg) and $\mathrm{Zn}^{2+}$ (OCBZn) in $10 \mathrm{mM}$ citrate-buffered solution at $\mathrm{pH} 4.5$ under stressed condition at a temperature of $70{ }^{\circ} \mathrm{C}$ for 5 days. Oxytocin recovery determined by LC-MS. The results are depicted as averages of three independent measurements $\pm \mathrm{SD}$.

Table 4. The Effect of Divalent Metal Ions on the Intensity of Degradation Products of Oxytocin Found after Stressing at $70{ }^{\circ} \mathrm{C}$ for 5 Days in $10 \mathrm{mM}$ Citrate Buffer ${ }^{a}$

\begin{tabular}{lccc} 
& \multicolumn{3}{c}{ rel peak intensity (\%) } \\
\cline { 2 - 4 } \multicolumn{1}{c}{ assignment } & OCBCa & OCBMg & OCBZn \\
\hline $\begin{array}{l}\text { oxytocin } N \text {-citryl amide } \\
\text { monodeamidation at Asn }{ }^{5} \text { or } \\
\quad \text { Gln }^{4}\end{array}$ & $71 \pm 19$ & $67 \pm 20$ & $52 \pm 12$ \\
oxytocin $N$-citryl imide & $58 \pm 86^{b}$ & $436 \pm 65^{b}$ & $906 \pm 50^{b}$ \\
oxytocin trisulfide & $40 \pm 39$ & $73 \pm 9$ & $50 \pm 8$ \\
oxytocin trisulfide $N$-citryl imide & $3 \pm 4$ & $4 \pm 5$ & $30 \pm 28$ \\
$N$-acetyl oxytocin ${ }^{c}$ & $107 \pm 7$ & $89 \pm 13$ & $114 \pm 13$ \\
oxytocin tetrasulfide & $53 \pm 64$ & $31 \pm 5$ & $33 \pm 31$ \\
dimer 1 & $5 \pm 1$ & $5 \pm 4$ & $2 \pm 1$ \\
dimer 2 & $7 \pm 1$ & $10 \pm 7$ & $3 \pm 2$ \\
dimer 3 & $15 \pm 11$ & $14 \pm 7$ & $6 \pm 4$ \\
dimer 4 & $172 \pm 42^{b}$ & $150 \pm 54^{b}$ & $90 \pm 7^{b}$ \\
dimer 5 & $15 \pm 11$ & $15 \pm 5$ & $6 \pm 5$ \\
dimer 6 & $27 \pm 21$ & $17 \pm 9$ & $17 \pm 16$ \\
dimer 7 & $43 \pm 42^{b}$ & $42 \pm 11^{b}$ & $31 \pm 30^{b}$
\end{tabular}

${ }^{a}$ The level of degradation products is expressed relative to the levels found in the solution without metal ions. ${ }^{b}$ Weak MS signal. ${ }^{c}$ No degradation product; compound was also found in the original oxytocin preparation.

The combination of citrate buffer and divalent metal ions greatly reduces the formation of most dimers after thermal stress. The absence of a significant decrease in the intensity of dimer 4 hardly contributes to the total amount of dimers because its intensity in the formulation without divalent metal ions after thermal stress was already low.

In this study, the buffer used was citrate, which is considered to be safe and occurs in many foods and is also a normal metabolite in the body. Its calcium, potassium and sodium salts do not constitute a significant hazard to humans. ${ }^{21}$ After thermal stress, covalent citrate-oxytocin adducts were formed through a mechanism involving the intermediate production of citrate anhydride, which reacts with the $N$-terminal amino group from the cysteine residue. ${ }^{14}$ Inhibition of the formation of $N$-citryl oxytocin by divalent metal ions as found in the present study might be due to a differential interaction of oxytocin and divalent metal ions for citrate. Wyttenbach et al. ${ }^{22}$ found that under acidic conditions ( $\mathrm{pH}$ 3.0) divalent metal ions bind to the carbonyl groups in the ring structure of oxytocin. The presence of doubly charged cations, such as $\mathrm{Zn}^{2+}, \mathrm{Mg}^{2+}$, $\mathrm{Ni}^{2+}, \mathrm{Mn}^{2+}$ and $\mathrm{Co}^{2+}$, has been found to be essential in increasing the potency of the specific binding of oxytocin to its receptor. Therefore the complex formed might increase the biological activity. ${ }^{23}$ Further, isothermal titration calorimetry data showed that citrate interacts with divalent metal ions, and this interaction is stronger than that of citrate with oxytocin (Tables SI and SII and Figures S21-S22 in the Supporting Information). Free citrate ions are probably more reactive toward the $\mathrm{N}$-terminal amino group from the cysteine residue than divalent metal-citrate adducts. In the presence of divalent metal ions, the formation of metal-citrate adducts reduces the concentration of free citrate, which reduces the driving force for citrate adduct formation with oxytocin. ${ }^{15}$ A second possibility is that binding of a divalent metal ion to oxytocin may change the position of the $\mathrm{N}$-terminal amino group from the cysteine residue rendering it less accessible for binding with citrate.

It can be concluded that citrate has two opposite effects on oxytocin stability. First, as also shown by Poole et al., ${ }^{14}$ it is reactive itself and can attack the $N$-terminal amino group from the cysteine residue to form an adduct. Second, as shown in our previous study, ${ }^{15}$ it protects oxytocin from degradation in the presence of divalent metal ions. In this study, we clearly show that the stabilization is due to the suppression of $N$-citryl oxytocin, tri/tetrasulfide and dimer formation. Furthermore, all reactions that were suppressed occurred on $\mathrm{Cys}^{1}$, and possibly $\mathrm{Cys}^{6}$. Cysteine is susceptible to oxidation and $\beta$-elimination, and degradation of oxytocin involving cysteine leads to dimerization, formation of tri/tetrasulfide and $\beta$-elimination followed by thio-ether formation.

Divalent metal ions in combination with citrate buffer suppress intermolecular reactions in the ring structure of oxytocin presumably by forming a complex in the region where the degradation reaction occurs. No significant difference was observed among the three tested divalent metal ions, $\mathrm{Ca}^{2+}, \mathrm{Mg}^{2+}$, and $\mathrm{Zn}^{2+}$, suggesting that divalency is the most important property of the metal contributing to stabilization of the oxytocin-metal-citrate cluster.

\section{ASSOCIATED CONTENT}

\section{S Supporting Information}

Figures depicting the MS/MS series of reduced oxytocin, numerous product ion spectra, and calorimetric titration profiles and tables of thermodynamics data. This material is available free of charge via the Internet at http://pubs.acs.org.

\section{AUTHOR INFORMATION}

\section{Corresponding Author}

*University of Groningen, Department of Pharmaceutical Technology \& Biopharmacy, Antonius Deusinglaan 1, 9713 AV Groningen, The Netherlands. Tel: 0031.50 3633172. Fax: 0031.50 3632500. E-mail: c.avanti@rug.nl.

\section{ACKNOWLEDGMENTS}

The authors want to thank MSD Oss for providing oxytocin for the study. This study was performed within the framework of the Dutch Top Institute Pharma project: number D6-202. 


\section{REFERENCES}

(1) Dale, H. H. The Action of Extracts of the Pituitary Body. Biochem. J. 1909, 4, 427-447.

(2) Karbiwnyk, C. M.; Faul, K. C.; Turnipseed, S. B.; Andersen, W. C.; Miller, K. E. Determination of oxytocin in a dilute IV solution by LC-MS(n). J. Pharm. Biomed. Anal. 2008, 48, 672-7.

(3) Owen, J.; Hauth, J. C. Oxytocin for the induction or augmentation of labor. Clin. Obstet. Gynecol. 1992, 35, 464-75.

(4) Chaibva, F. A.; Walker, R. B. Development and validation of a stability-indicating analytical method for the quantitation of oxytocin in pharmaceutical dosage forms. J. Pharm. Biomed. Anal. 2007, 43, 179-85.

(5) Gard, J. W.; Alexander, J. M.; Bawdon, R. E.; Albrecht, J. T. Oxytocin preparation stability in several common obstetric intravenous solutions. Am. J. Obstet. Gynecol. 2002, 186, 496-8.

(6) Turner, R. A.; Pierce, J. G.; du Vigneaud, V. The purification and the amino acid content of vasopressin preparations. J. Biol. Chem. 1951, 191, 21-28.

(7) Davoll, H.; Turner, R. A.; Pierce, J. G.; du Vigneaud, V. An investigation of the free amino groups on oxytocin and desulfurized oxytocin preparations. J. Biol. Chem. 1951, 193, 363-370.

(8) Winkler, M.; Rath, W. A risk-benefit assessment of oxytocics in obstetric practice. Drug. Saf. 1999, 20, 323-45.

(9) du Vigneaud, V; Ressler, C; Trippett, S. The sequence of amino acids in oxytocin, with a proposal for the structure of oxytocin. J. Biol. Chem. 1953, 205, 949-957.

(10) Ananthanarayanan, V. S.; Brimble, K. S. Interaction of oxytocin with $\mathrm{Ca}^{2+}: \mathrm{I} . \mathrm{CD}$ and fluorescence spectral characterization and comparison with vasopressin. Biopolymers 1996, 40, 433-43.

(11) Gimpl, G.; Fahrenholz, F. The oxytocin receptor system: structure, function, and regulation. Physiol. Rev. 2001, 81, 629-83.

(12) Hogerzeil, H. V.; Walker, G. J. A.; De Goeje, M. J. Stability of injectable oxytocics in tropical climates; World Health Organization: Geneva, 1993; WHO/DAP/93.6.

(13) Hawe, A.; Poole, R; Romeijn, S.; Kasper, P.; van der Heijden, R.; Jiskoot, W. Towards heat-stable oxytocin formulations: analysis of degradation kinetics and identification of degradation products. Pharm. Res. 2009, 26, 1679-88.

(14) Poole, R. A.; Kasper, P. T.; Jiskoot, W. Formation of amide- and imide-linked degradation products between the peptide drug oxytocin and citrate in citrate-buffered formulations. J. Pharm. Sci. 2011, 100, 3018-3022.

(15) Avanti, C.; Amorij, J. P.; Setyaningsih, D.; Hawe, A.; Jiskoot, W.; Visser, J.; Kedrov, A.; Driessen, A. J.; Hinrichs, W. L.; Frijlink, H. W. A new strategy to stabilize oxytocin in aqueous solutions: I. The effects of divalent metal ions and citrate buffer. AAPS J. 2011, 13, 284-290.

(16) The United States Pharmacopeial Convention, Rockville, MD, 2005; USP-29 NF-24.

(17) Shaw, G. S. Synthetic calcium-binding peptides. Methods Mol. Biol. 2002, 173, 175-82.

(18) Huck, C. W.; Pezzei, V.; Schmitz, T.; Bonn, G. K.; BernkopSchnurch, A. Oral peptide delivery: are there remarkable effects on drugs through sulfhydryl conjugation? J. Drug Targeting 2006, 14, $117-25$.

(19) Manning, M. C.; Chou, D. K.; Murphy, B. M.; Payne, R. W.; Katayama, D. S. Stability of protein pharmaceuticals: an update. Pharm. Res. 2010, 27, 544-75.

(20) Bulaj, G. Formation of disulfide bonds in proteins and peptides. Biotechnol. Adv. 2005, 23, 87-92.

(21) Joint FAO/WHO Expert Committee on Food Additives. Citric acid and its calcium, potassium and sodium salts; 1974; Vol. 539.

(22) Wyttenbach, T.; Liu, D.; Bowers, M. T. Interactions of the hormone oxytocin with divalent metal ions. J. Am. Chem. Soc. 2008, 130, 5993-6000.

(23) Pearlmutter, A. F.; Soloff, M. S. Characterization of the metal ion requirement for oxytocin-receptor interaction in rat mammary gland membranes. J. Biol. Chem. 1979, 254, 3899-906. 